\title{
Experimentelle Studien über die fettige Entartung des Muskelgewebes.
}

\author{
Von
}

\section{Alexander Stuart}

aus Petersburg.

Hierzu Taf. XXV.

Wie bekannt gehört die Muskelentartung nicht zu den acuten Krankheiten, deren erste Entwicklung im Organismus durch heftige Rückwirkungen auf andere Organsysteme begleitet wäre; - daher wird sie durch klinische Untersuchung nur in ihren vorgerückten Stadien nachgewiesen, was zur Folge hat, dass die Entartung hauptsächlich in ihren entwickelteren, späteren Formen untersucht wird, wie in den seltenen Fällen, wo sie die Ursache des Todes abgiebt, so z. B. in den bekannten Meryan'schen Fällen, oder in dem Falle, wo ein Individuum mit noch am Leben klinisch nachgewiesener Entartung, durch diese und andere Störungen zu Grunde ging; oder wo, was am häufigsten geschieht, das makroskopische Aussehen der Muskeln einer secirten Leiche, die Veranlassung zu der mikroskopischen Untersuchung giebt. In allen diesen Fällen hat es der Forscher nur mit der schon ausgeprägten entwickelten Krankheit zu thun; die ersten Stadien der Entartung sind so schwierig zu verfolgen, die neugebildeten Elemente so den normalen ähnlich, dass er sie allenfalls nur als Leichenerscheinungen deuten würde, wenn er sie überhaupt wahrnähme, und sich eher an die sicherere, mehr ausgeprägte Erscheinung halten würde, die schon den späteren Stadien angehört und in der Weise bleiben ihm die ersten Entwicklungs- 
stufen verborgen, und werden ihm möglicherweise verborgen bleiben, wollte er auch, mit dem speciellen Zwecke den krankhaften Vorgang von Anfang an zu verfolgen, die verschiedenen ihm zu Gebote stehenden Leichen darauf untersuchen, denn obgleich er die letzten Stadien des Vorganges auch kennt, darf er doch nicht alle möglichen von ihm beobachteten Veränderungen als Glieder oder Abstufungen der Entwickelung des ihm schon früher bekannten Zustandes auffassen.

Wenn für andere Gewebe und Organe diese Schwierigkeiten besiegbare sind, so bietet die Aehnlichkeit der veränderten Elemente des Muske]gewebes mit den normalen solche Schwierigkeiten bei der Untersuchung, dass die genaue Verfolgung des Entwickelungsganges des besagten Prozesses auf rein anatomischem Wege fast unmöglich ist, selbst hei der grössten darauf verwendeten Mühe und Zeit, während auf dem experimentellen Wege es sehr leicht gelingt durch Parallelversuche zum Ziele zu kommen, was dadurch noch besonderen Werth erlangt. dass eine künstliche Hervorbringung der Krankheit bei Thieren selır leicht gelingt. Alle diese Unstände haben mich bewogen, als ich bei Gelegenheit vergleichend-histologischer Studien über die normilen Muskeln und über die anderen contractilen Gewebe, die mich seit einiger Zeit beschäftigen, meine Aufinerksamkeit den pathologischen Vorgängen in den Muskeln zuwendete, zu der experimentellen Methode überzugehen.

Die klinischen Erfahrungen zeigten gewissermaassen den Weg an, den man einhalten solle, um bei Thieren künstliche Zustände hervorzurufen, welche denen ähnlich wären die die vorher beobachteten Entartungserscheinungen in den Muskeln des Menschen bedingen. Die Mehrzahl der Autoren stimmt darin überein, dass die Muskelentartung mit einer andauernden Entzündung hervortritt, ich musste daher bei meinen Versuchen mir zur Aufgabe stellen eine solche hervorzubringen. Mechanische Verletzungen und Reize der Muskeln, Betupfung mit Säuren, Zinkpaste, Aetzkali und dergleichen hatten zu schwache oder langsame Wirkung, oder aber griffen die Gewebe zu stark an; ausserdem hatten sie alle die üble Folge, dass der Reiz nicht auf einer Stelle concentrirt blieb, sondern die unmittelbar zerstörende Wirkung des Reizmittels verbreitete sich ringsumher, z. B. bei Anwendung von Flüssigkeiten, was eine scharfe Localisirung des Reizes unmöglich machte. Ich zog daher die Aetzung mit Argentum nitricum vor, ein Verfahren, das mir ein Mittel in die Hand gab, die Lage, Stärke und Ausdehnung des Reizes je nach 
Bedürfniss ändern zu können. Die Versuche wurden an verschiedenen Thieren ausgeführt: an Kaninchen, Ratten, Tritonen, Fröschen, Krebsen, Käfern u. s. w. Bei den ersteren war die Resorption der entarteten Bestandtheile sehr lebhaft, was mich nöthigte, starke und wiederholte Reize anzuwenden; bei Krebsen und Küfern.wurden nur die ersten Stadien der Degeneration erzielt, ausserdem starben die Thiere bald. Ratten und Tritonen boten weniger Bequemlichkeit wegen ihrer Aufbewahrung, die letzteren auch wegen der Kleinheit ihrer Muskeln; am hesten bewährte sich der Frosch, an dem auch der grösste Theil der Versuche angestellt wurde; die unten mitzutheilenden Resultate beziehen sich aber auch auf die drei erstgenannten Thierarten, die ganz dieselben Erscheinungen darboten. Die Aetzung wurde nach der Durchschneidung der Haut ganz oberflächlich und kurz andauernd ausgeführt, damit eine Muskelschicht von nicht mehr als $1 / 2$ Quadratcentimeter Breite und $1 \mathrm{~mm}$. Tiefe direkt der Wirkung des Aetzmittels ausgesetzt. sei. Wegen der Dünnheit der Fascie war bei diesen Thieren das Einschneiden des Muskels unnöthig, besonders da eine Aetzung, die tiefer in den Muskel eingreift, zu nahe an den Nervenstamm reicht, was immer gefährliche Folgen nach sich zieht. Deswegen wurden die Muskeln des Oberschenkels, als die dicksten, gewählt; andererseits macht ihre grössere Entfernung von den edlen Organen und grossen Nervencentren die Aetzung minder gefährlich, so das auf diese Weise geätzte Frösche, besonders die wenig reizbaren Winterfrösche, monatelang erhalten blieben, was mir auch gestattete durch wiederholte Ausschneidungen den Fortgang des krankhaften Prozesses in einem und demselben Muskel vollkommen $\mathrm{zu}$ verfolgen.

Obgleich die Literatur über unseren Gegenstand sehr reich ist, so sind dadurch, dass viele Autoren sich nicht deutlich darüber ausgesprochen haben, welcher Ansicht über die Structur der Muskeln sie huldigen, viele an sich ganz richtige Beobachtungen nicht in ihrem vollen Maasse zu verwerthen; denn, wenn wir aus diesen erfahren, wie der veränderte Muskel beschaffen ist, so wissen wir doch nicht, aus welchen Elementen des normalen Gewebes die verschiedenen Elemente des pathologischen hervorgegangen sind. - Ich will hier nur kurz erwähnen, dass ich der Ansicht bin, dass die Fibrille die letzte (das heisst, soweit als unsere Analyse bis jetzt ausreicht), physiologische Einheit des Muskels ist, was für die verschiedenen Thierklassen zu beweisen ich anderwärts versuchen werde. Als 
Typus der Fibrille muss ich perlschnurartig aneinandergereihte Reihen von Fleischtheilchen (sarcous elements Bowman's) von verschiedener Form, rund, oval, parallelepipedon ähnlich, mit abgerundeten Ecken (bei Arthropoden) u.s.w. annehmen, von denen jede Reihe in Protoplasmamasse eingehüllt ist, die die eigentliche contractile Substanz des Muskels abgiebt. ${ }^{1}$ ) (Fig. 1.)

Was die pathologischen Veränderungen selbst betrifft, so müssen wir bei der von uns vorgenommenen Aetzung mit Arg. nitricum zwei Arten unterscheiden, das heisst die, welche durch die rein chemische Wirkung dieses Aetzmittels auf die Muskelsubstanz bedingt sind,

1) In der Zeitschrift für wiss. Zoologie XV. Bd. Taf. VII Fig. 11 c. gab ich eine balb schematische Darstellung der Fibrille einer Aplysialarve. Die Existenz einer lichten, die Fleischtheilchen auch seitlich umgebenden Substanz gehört zu den schwierigsten micographischen Aufgaben, indem ihr schwacher Brechungscoefficient in Verbindung mit ihrem geringen Durchmesser diesen Nachweis nur durch ausserordentlich penetrirende, dabei aber gut definirende Linsen gestattet, Eigenschafton, die nur in den wenigsten Linsen vereinigt sich finden. Carpenter The microscope, pag. 730, Fig. 376 giebt ein mit starken englischen Linsen gewonnenes Bild einer Säugethierfibrille, in welchem diese seitliche Contour mit aller Bestimmtheit angegeben ist, aber durch übertriebene cellulare Ansichten verleitet, nimmt er an, dass jedes Fleischtheilchen mit der ihn allerseits umgebeuden lichten Substanz eine Zelle sei. wobei diese seitliche, nebst einer medianen die Fleischtheilchen von einander trennenden Contour, die Contour der Zellenmembran sei, was keineswegs der Fall ist, da die seitliche Contour eine ebene, durch die ganze Länge der Fibrille ununterbrochen fortlaufende ist, die auch gar nicht seitlich von der Berührungsfläche der beiden Fleischtheilchen bis zu ihrer Begegnung mit den seitlichen Contouren 'verfolgt werden kann, was im $\mathrm{C}$ ar penter'schen Sinne jedenfalls der F'all sein sollte. Ein sehr geeignetes Object bildet das bekannte, von Powell u. Lealand gelieferte Schweinmuskelpräparat, an welchem, als ich noch für keine der concurrirenden Ansichten mich entscheiden konnte, Prof. Schiff in Florenz mir auf eine höchst sinnreiche Weise mit Hülfe des an ihn übrgangenen Arbeitsmikroskops von Amici, den strengen optischen Beweis lieferte für die wirkiiche Existenz dieser seitlichen Contour, somit auch der die Fleischtheilchenreihe umgebenden hellen Substanz, nebst einigen interessanten, leider bis jetzt noch nicht publicirten Einzelnheiten über die Fleischtheilchen einiger Säugethiere. Ich kann diese Verhältnisse mit geübtem Ange, mit meinem Immersionssysteme Nro. 10 von $\mathrm{Hartnack}$, oder mit der Linse $F$ von $Z$ eiss, schon deullich erkennen, viel schärfer aber mit einer an Stärke zwischen Nro. 9 und 10 stehenden Oelimmersionslinse von Amici, die durch die Gefälligkeit von Prof. Schiff nebst anderen schwächeren, mir aus dem Nachlasse von Amici zugekommen ist, und die zu den besten gehört, die er geliefert hat und zwischen seinen eigenen Arbeitslinsen staud. 
und die welche das Produkt der durch die Entzündung hervorgerufenen pathologisch-physiologischen Thätigkeit sind.

Nach vollzogener Aetzung nimmt eine je nach dem Grade der Aetzung mehr oder minder dicke Schicht der Muskelsubstanz (bei unseren Versuchen gewöhnlich $1 / 2-1 \mathrm{Mm}$.) eine diaphane weisse Farbe an, wie es auch andere eiweisshaltige Gewebe bei solcher Aetzung thun. Bei näherer Untersuchung ergiebt sich, dass das Muskelgewebe in hohem Grade verändert ist. Nicht nur jede regelmässige, die Querstreifung bedingende Anordnung der Fleischtheilchen wird vermisst, sondern die Muskelfaser bildet einen Schlauch, der mit einer feinkörnigen, theilweise feinfaserigen Detritusmasse von blasser milchweisser Farbe, die nur als ein Coagulationsprodukt der Muskelsubstanz zu betrachten ist, ausgefüllt erscheint (Fig. 2): Das chemische Verhalten ist das der coagulirten Albuminate: Unlöslichkeit in Wasser, Alkohol, Aether, Essig- und Salzsäure und nicht zu starken Aetzkalilösungen. In der gesunden Faser sieht man, dass einzelne Fibrillenbündel sich enger aneinanderschliessen als an den übrigen, was eine Art Spaltung der Faser in eine Anzahl Fibrillenbündel bedingt; diese Spaltung wird in dem in Detritus zerfallenen Muskel, durch Scheidung der Detritusmasse, in gewisse Längsabtheilungen noch erhalten, wobei die Detritusmasse wie langfaserig erscheint, was darauf hinzuweisen scheint, dass diese Längsabtheilungen von einander durch irgend welche Binde- oder Scheidemittel getrennt sind, über deren Natur wegen ihrer allzugeringen räumlichen Ausdehnung natürlich sich nur Vermuthungen anstellen lassen, auf welche wir uns aber nicht einlassen wollen. Diese Längstheilungen sind sehr blass und lassen sich nur an einzelnen Stellen verfolgen; man kann sie in den veränderten Fasern nur in den noch ganz glatt gebliebenen verfolgen, mit der Wulstbildung wird die ganze Masse stark gepresst und die Scheidung hört auf.

Das Sarcolemma nimmt an Festigkeit ein wenig ab; die darin enthaltene Masse ist anfänglich sehr weich, und kann daher in diesen Zustande verschiedene, den äusseren Umständen entsprechende Formen annehmen, in welchen sie bald durch Festwerden fixirt wird ; durch die durch Aetzung hervorgerufenen lebhaften Contractionen der unterliegenden Muskelschichten werden diese veränderten Fasern hin nnd her gezerrt und gedrückt, dadurch wird die sie ausfüllende weiche Masse zu unregelmässigen, nierenförmig abgerundeten Ballen und Wülsten geformt (Fig. 2 a), und übt daher einen 
ungleichmässigen Druck auf das Sarcolemma, das einerseits durch die herausragenden Wülste stärker gedehnt wird, andererseits die durch Einschnürungen der Detritusmasse freigelassenen Rüume auszufüllen strebt, was zuerst zu Faltenbildung Anlass giebt, dann wenn durch die Zusammenziehungen des Muskels die Spannungsunterschiede der verschiedenen Stellen vergrössert werden, auch zu Querrissen führt. Bei relativ schwächeren Aetzungen ist die unthätig gewordene geätzte Schichte dem Drucke und Zuge des unterliegenden Muskels, seiner Dünnheit wegen, im hohen Grade ausgesetzt, was zur Folge hat, dass Fasern mit glattem, nicht ballenförmig zusammengepresstem Inhalte, eine verschwindend kleine Zahl bilden, und je länger die Aetzung fortbesteht, desto mehr nimmt die Zahl der Risse des jetzt seine ursprüngliche Elasticität verlierenden Sarcolemma's zu, die nach Belieben bei unvorsichtiger Präparation noch vermehrt werden können. Erreicht die Dicke der unmittelbar geätzten Schichte etwa $2 \mathrm{Mm}$., so bildet sie eine Art Panzer um den Muskel, dessen Kraft ausreicht um ihn zu bewegen, aber nicht dazu, um ihn so $\mathrm{zu}$ beugen und zu zerren, wie es mit dünneren Schichten geschieht. Diese Veränderungen werden streng localisirt auf die von dem Aetzmittel direkt betroffenen Fasern, und wenn es nicht etwa durch das in die Wunde hinfliessende Blut in weitere Regionen fortgeführt wird, so sind nur diese Fasern und von ihnen blos die einzelnen direkt betroffenen Theile in die Veränderung hineingezogen, die übrigen Theile aber der körnigen Entartung verfallen.

Die Muskelfasern, die unter dieser Detritusschicht liegen und welche von dem Aetzmittel nicht direkt betroffen wurden, zeigen ganz anderes Verhalten.

Sie werden der Sitz einer durchgreifenden Veränderung, die verschiedene Entwickelungsstufen durchmachend, als endliches Resultat die Umwandlung der Proteinsubstanz der Muskelfaser in Fett ergiebt.

Einige Autoren haben die Muskelatrophie als ein allmähliges Schwinden der Muskelsubstanz unter dem Drucke eines üppigen Nachwuchses von sich aus dem Bindegewebe entwickelnden Zellen betrachtet (Billroth, Böttcher). Ich muss aber als Sitz des pathologischen Vorganges das Muskelgewebe selbst bezeichnen, und wenn auch das Auftreten neugebildeter Zellen im Bindegewebe beobachtet wurde, so war es in einem nur untergeordneten Grade, 
Experimentelle Studien über die fettige Entartung des Muskelgewebes. $\mathbf{4 2 1}$

wobei dieses Auftreten keineswegs als bedingende Ursache der Entartung angesehen werden konnte.

Die erste Veränderung, die in der Muskelfaser vor dem $\mathrm{Zu}$ standekommen von formellen Umbildungen wahrgenommen wird, betrifft die allgemein physikalischen Verhältnisse der Muskelfaser als Ganzes.

Sie bietet eine zähe, elastische, glasartig durchsichtige Masse dar, die in Essig- und Phosphorsäure unlöslich, in Alkohol und nach Erhitzung über $50^{\circ}$ coagulirend, in Aether nicht coagulirbar ist. Schwefelsäure bewirkt einen schwach gelblichen Niederschlag; Salpetersäure eine lebhafte Xanthoproteinsäurereaction ; durch Salzsäure wird die Masse erweicht.

Die Querstreifung erscheint sehr blass und verwaschen, dabei erscheint sie am bestimmtesten auf der Oberfläche der Faser, be tieferer Focaleinstellung in dickeren Fasern scheint sie manchmal ganz zu fehlen, die Farbe der Faser ist eine durchsichtig weissliche, ins bläuliche übergehend und ein wenig opalescirend. Alle diese Umstände deuten auf eine Veränderung der chemischen Zusammensetzung der Faser, dabei deuten die oben angegebenen Reactionen darauf hin, dass diese Veränderung im Sinne einer Annäherung zu der Zusammensetzung des gewöhnlichen Albumins (Serunalbumin?) vor sich gegangen ist. Da diese Reactionen nur auf microchemischem Wege gewonnen wurden, so können sie selbstverständlich nur auf eine solchen zukommende Genauigkeit Ansprüche machen. Solches Blasswerden der Muskelfaser wurde vielfach beobachtet (Virc how Böttcher, Oppenheimer, O. Weber), meistens aber neben anderen Veränderungen, oder als selbstständige "glasartige» Entartung aufgeführt, weniger aber als Ausgangspunkt jeder weiteren Entartung betrachtet, wofür wir es jedenfalls halten müssen.

Dieses Blasswerden betrifft. die Fleischtheilchen wie die Umhül-. lungsmasse, vorläufig aber ist auch mit den besten Linsen keine andere Veränderung der bestehenden und keine Herausbildung neuer constituirender Elemente zu bernerken.

Bei der weiteren Verfolgung des Entartungsprocesses wird mangewöhnlich zuerst auf das Auftreten der sogenannten minterstitiellen « Körnchen aufmerksam, die wegen ihrer Grösse und ihres starken Brechungsvermögens zuerst bemerkt werden, die aber nicht die Anfänge des Entartungsprocesses darstellen, sondern das zuerst deutlich hervortretende Stadium desselben. 
Parallel mit dem Blasswerden der Faser geht eine tiefgreifende morphologische Veränderung derselben vor sich, indem die Bestandtheile der Fibrille (Fleischtheilchen und umhüllende contractile Substanz), die vorhin als homogene, nur verschieden dichte Substanzen sich darboten, jetzt in moleculäre Massen sich differenziren, deren einzelne Molecüle auf der Grenze des Wahrnehmbaren stehen; obgleich dieser Vorgang in an sich selbst schon sehr feinen Theilen statt hat, ist er doch mit hinreichend feinen Mitteln und Methoden deutlich wahrnehmbar.

Durch Zusammentliessen dieser Molekeln werden grössere, an Umfang sich den Fleischtheilchen nähernde Körnchen gebildet. Ihre Lage, vorzugsweise in Längsreihen, wird durch die Organisationsverhältnisse der Muskelfaser bedingt; da die die Zwischenräume der Fleischtheilchen einnehmende contractile Substanz weniger dicht ist als letztere, so lagern sich die neugeformten Körnchen vorzugsweise in derselben ein, besonders aber auf den Stellen, wo die oben beschriebenen Zwischenräume der Fibrillenbündel sich befinden, und da die seitlichen $Z$ wischenräume grösser als die verticalen sind, so ist auch eine solche Lagerung, noch mehr aber in den Räumen zwischen je vier Fleischtheilchen, die natürlichste, womit auch das auf mehreren Stellen der Faser immer vorkommende Aneinanderreihen der Degenerationskörner leicht erklärlich wird. In dieser Weise schreitet die Entartung immer weiter fort, die Körnerablagerung wird nicht gleichmässig in allen Theilen der Faser vollzogen, sie geht vielmehr von mehreren Centren aus, sich stärker in Längs- (das heisst in der Richtung der Muskelfaser) als in der Querrichtung ausbreitend; als Resultat dieser Thätigkeit erscheinen in verschiedenen Abständen von einander stehende, parallelverlaufende, unterbrochene Reihen perlschnurartig aneinandergereihten Körner; zu derselben Zeit ist aber die pathische Thätigkeit auch in der Querrichtung, wenngleich viel schwächer wirksam; da aber die ursprüngliche Textur der Muskelfaser durch die vorhergegangenen Veränderungen geschwächt wurde, so sind die Bahnen zu den Ansammlungsorten der ausgeschiedenen Molekeln nicht mehr so scharf bezeichnet wie früher, was zur unmittelbaren Folge hat, dass die jetzt gebildeten Körner nicht mehr in derselben regelmässigen Ordnung, wie es früher geschah, abgelagert werden; vielmehr bildet sich eine grössere Anzahl kleinerer, in Unordnung oder haufenweise liegender Körner; ausserdem bleiben noch zwischen den gebildeten Körnchen einzelne Partien nicht geformter Volecularmasse. 
Je mehr Thätigkeitscentren zu derselben Zeit in einer Stelle der Faser entstanden sind, desto regelmässiger sind die Reihen der neugebildeten Körner; je allgemeiner und allmähliger die Veränderung eingetreten ist, desto unregelmässiger in Grösse und Anordnung sind dieselben. Durch ihre Form und Grösse unterscheiden sich die Entartungskörner nur sehr wenig von den normalen Fleischtheilchen; desto grösser aber ist der Unterschied in ihren allgemeinphysikalischen Eigenschaften. Die Entartungskörner haben einen viel geringeren Brechungscoefficienten als der der Fleischtheilchen ist, derselbe scheint zwischen dem Brechungscoefficienten der contractilen Substanz und dem derFleischtheilchen zu stehen. Diese Verminderung des Brechungscoefficienten ist durch die geringe Dichtigkeit des entarteten Gewebes bedingt, und da bei der Entwickelung des Entartungsprocesses kein Substanzverlust (den Substanzverlust durch die physiologische Leistung nicht mitgerechnet, der aber in diesem Falle in der Gefangenschaft höchst unbedeutend ist) zu bemerken ist, so kann die bei solchen Entartungen wahrnehmbare Dickenzunahme des Muskelbündels dadurch erklärt werden, dass die in Sarcolemmasacke enthaltene Masse durch die Verminderung ihrer inneren Dichtigkeit gezwungen ist sich im Raume mehr auszubreiten, wozu ihr die Verminderung der Widerstandsfähigkeit des Sarcolemma's auch die beste Gelegenheit giebt.

Ausserdem zeigen die Entartungskörner gar keine Spuren der für die Fleischtheilchen so charakteristischen Doppelbrechung. Diese in so hohem Grade merkwürdigen Eigenschaften dieser Körner zeigen uns, dass der Entartungsprocess nicht nur in Veränderungen der formellen Bestandtheile des Muskelgewebes besteht, sondern vielmehr ihm eine tiefe Umgestaltung in der molecularen Beschaffenheit des Gewebes zu Grunde liegt; eine Thatsache, die von unverkennbarer Tragweite für die Auffassung des krankhaften Vorganges und dessen weitere Entwickelung ist ${ }^{1}$ ).

In diesem Stadium der Entartung bietet das Sarcolemma auffallende Veränderungen, die in einer bedeutenden Verdickung, in

1) Aus diesem Beispiele sehen wir wieder, von welcher Wichtıgkeit die Anwendung des polarisirten Lichtes ist in allen den Fällen, wo es sich darum handelt, Aufschlüsse über die innere moleculare Structur eines organischen Körpers zu erhalten. 
einem Verlust der ursprünglichen Derbheit und Opakwerden bestehen, was auf eine Durchtränkung der Membran mit der sich jetzt in der Muskelfaser ansammelnden Exsudattlüssigkeit zurückgeführt werden kann. Seine Elasticität ist aber noch so gross, dass die Contractionen der Vuskelfasern nicht zu solchen Rupturen führen, wie es im vorigen Falle war. Bei unaufmerksamer Beobachtung erscheint das Sarcolemma durch angeklebte Exsudatflüssigkeit und feine Entartungskörner noch dicker und mit rauher innerer Oberfläche. was natürlich nur eine Täuschung ist.

Die Muskelkörperchen vergrössern sich in einem ansehnlichen Maasse, behalten aber ihre Zusammensetzung; das Blasswerden der Uuskelfaser erlaubt es, sie im frischen Zustande auf sehr genaue Weise zu beobachten, wobei man sich dentlich vom Mangel der ihnen vielfach zugeschriebenen Membran überzergen kann. Vermehrung derselben kommt nur in einer viel späteren Zeit und anch in weit geringerem Maasse vor, als es von Vielen angenommen wird.

In einem solchen Zustande trifft man die Muskelfaser 2-3 Wochen nach der Aetzung. In den folgenden Wochen unterliegen die Entartungskörner einer noch durchgreifenderen Veründerung, indem ihre Albuminmasse sich in Fett um andelt. Zuerst ist diese Cmwandhung nur vereinzelt, nach und nach aber greift sie stärker um sich, bis der überwiegendste Theil der Muskelmasse sich in Fett verwandelt hat, was je nach Umständen manchmal schon in 8 Wochen, gewöhnlich aber nicht vor 3 Monaten geschieht. Da die Umwandlung eine allmählige ist, so lässt sich ein bestimmter Zeitpunkt überhaupt nicht angeben; die Dauer der Entartung hängt ausserdem von der Stärke der Aetzung und der Jahreszeit ab; in Allgemeinen haiten die so reizbaren Frühlingsfrösche starke Aetzungen und Gefangenschaft nicht lange aus; die beim Anfange meiner Versuche angewandten Frösche hielten sich nicht sehr gut, da sie schon sehr abgespannt durch die lange Wintergefangenschaft waren und bei der strengen Kälte (November) es immer schwieriger war ihnen eine geeignete gleichmässige Temperatur zu verschaffen. Das durch die Umwandlung der Entartungskörner gebildete Fett erscheint zuerst in Form von kleinen Tröpfchen, die mit den nebenan liegenden sich zu vereinigen streben, was zur Folge die Bildung grösserer Fetttropfen hat; vor oder nach dieser Vereinigung bilden sich un diese Fetttröpfchen, durch Füllung der sie umgebenden albuminoiden Exsudattlüssigkeit, ganz feine, durchsichtige Häut- 
chen. eine Erscheinung, die überall beim Zusammentreffen von Fett und Albuminsubstanzen stattfindet und die, wie bekannt, von A s c h erson auch experimentell erzeugt wurde. Die so umhäuteten Fetttropfen können sich nicht mehr miteinander vereinigen, bis durch änsseren Druck ihre Häute zum Platzen gebracht werden, was auch vielfach geschieht. Die Häutchen können bei unvollständiger Ausziehung mit Aether auf sehr zierliche Weise dargestellt werden, indem sie dabei schrumpfen und Falten bilden.

In der Weise wird es leicht erklärlich. warum die Grösse und Lagerung der Fetttröpfchen so verschieden ist; zuerst sieht man nur ganz kleine zerstreut liegende Kügelchen, später findet man öfters die von Virchow hervorgehobenen Reihen perlschnurartig aneinandergereihter, ovaler Fetttröpfchen; zu dieser Gattung gehören die $\mathrm{K}$ ö1li k er'schen »interstitiellen Körnchen «, die bei lange unthätigen Muskeln sich immer bilden; in den späteren Stadien fliessen die kleineren Fetttröpfchen in grössere zusammen, die dann wieder die runde Form annehmen, da $\mathrm{zu}$ der Zeit der innere Zusammenhang der Bestandtheile der Muskelfaser derartig gelockert ist, dass der neugebildete Tropfen keinen Druckeinwirkungen ausgesetzt wird. Man darf diese (oft $1 / 2-1 \mathrm{Mm}$.) grossen Blasen durchaus nicht mit den in dem Bindegewebe des Muskelbündels bei längerer Unthätigkeit und reichlicher Nahrung sich entwickelnden Fettzellen verwechseln. Diese entwickeln sich ausserhalb der Muskelfaser aus von aussen zugebrachtem Materiale, jene dagegen in der Faser aus dem umgebildeten Faserinhalte. Böt tch e'r hat ganz Recht. wenn er sagt, dass wir weitere Aufschlüsse über die Bjldung von Fett in den Muskeln nur durch das Mikroskop erhalten können, denn z. B. in den beiden hier angeführten Fällen wird die chemische Analyse uns dieselben Resultate geben, es ist aber einleuchtend, wie wesentlich verschieden die beiden Processe sind. Aus allem oben Mitgetheilten geht hervor, dass wir eine einfache Umbildung des albuminoiden Muskelfaserinhaltes in Fett anzunehmen haben; diesen Fettinhalt nur auf gesteigerte Zufuhr von Fett zu reduciren, wie es von Einigen geschehen ist, haben wir gar keine Veranlassung. Die Zufuhr geschieht wohl, aber dann manifestirt sie sich durch die oben berührten $\mathrm{Ab}$ lagerungen von Fettzellen in Bindegewebe; sie könnte auch im Muskelinhalte selbst geschehen, aber wir können es nicht wahrnehmen, daher eine solche Annahme Angesichts einer mehr naturgemässen und unmittelbar wahrzunehmenden als nicht nothwendig erscheint. 
Die Untersuchung der durch Auspressen gewonnenen Fette und des Aetherextractes ergab, dass wenn auch Beimischungen von Palmitin und Stearin wahrscheinlich sind, so besteht es hauptsächlich aus Oleïn. Dieselben Erscheinungen, welche in unserem Falle durch Aetzungsreiz in der Muskelfaser hervorgebracht wurden, können auch durch den Reiz, den die Anwesenheit parasitischer Nematoden bedingt, zu Tage treten. In den von mir untersuchten Muskeln, die durch Trichinen (Kaninchen) oder den Myoryctes Weismanni Eberth, (Frosch, Triton) inficirt waren, war der Gang des Entartungsprozesses durch die bedeutendere Stärke des Reizes viel heftiger und schneller; ausserdem wurde durch die beständigen Bewegungen des Thieres die regelmässige Ausbildung grösserer Körner gehindert, daher auch der Muskel ball mit kleinen, rundlichen, gleichgrossen, starklichtbrechenden Körnern ausgefüllt erscheint. Die Verhältnisse bleiben aber im allgemeinen dort wie hier absolut dieselben, obgleich in letztem Falle die Erscheinung nicht so prägnant erscheint, da ihr Entwickelungsgang ein vielfach gestörter ist. In beiden Fällen kann es leicht zur Resorption und Ausgleichung des gebildeten Fettes kommen; so ist der von den Trichinen ausgeübte Reiz, obgleich sehr stark doch nicht sehr lange andauernd, da er nach der Einkapselung der Trichine grösstentheils aufhört; ebenso, wenn der Aetzungsreiz kein starker und oft wiederholter ist, kann es zur Ausgleichung koinmen; rorausgesetzt dass die Fettumbildung nicht zu weit um sich gegriffen hat. Ueberhaupt ist die fettige Entartung der Muskeln in ihren mittleren Stadien dem Organismus nur insofern unmittelbar schädlich, als sie durch die dadurch bedingte Lähmung der Bewegungen mittelbar eingreifend auf die Thätigkeit lebenswichtiger Organe wirkt. Aus der Vergleichung der oben angeführten Beschreibung des Entartungsvorganges mit den bis jetzt von den Autoren gemachten Angaben ersieht man, dass dieselben hauptsächlich die letzten Stadien der Fntartung, wenn die fettige Umwandlung schon vorgeschritten ist, betreffen, ohne die ersteren $z \mathfrak{z}$ berühren. Die fortgeschritteneren Grade der Entartung werden durch eine beträchtliche Volumabnahme des ganzen Muskels begleitet, die in einer Resorption und Ausscheidung des neugebildeten Fettes ihren Grund findet.

Es scheint, dass bei mässigeren Graden der Entartung der Schaden ein leicht ausgleichbarer ist, wobei die Muskelkörperchen sicherlich eine wichtige Rolle zu spielen haben, worauf man aus ih- 
rem Wachsthume und ihrer wiederholten Theilung zu schliessen hat; eine Thätigkeit, deren Endresultat ebenso wie die Zustände der Faser bei sehr starker Entartung nach geschehener Fettresorption ich leider in $\mathrm{zu}$ geringem Maassstabe zu beobachten Gelegenheit hatte, als dass ich darüber sichere Wittheilungen machen könnte. Ich will nur bemerken, dass die Fettmetamorphose auch in den Muskelkörperchen parallel mit der des Faserinhalts vor sich geht, so dass sie keineswegs die Centren sind, in welchen die Entartung zuerst entsteht, wie es mehrfach angenommen wurde, in ihnen ist freilich jede Veränderung viel leichter zu sehen als im Parenchym, besonders wenn man dickere Fasern untersucht.

Dass bei dem Aetzungsreize die Entartung eine viel leichter ausgleichbare ist als die in verschiedenen Krankheiten entstandene, wo eine solche auch durch eine Reihe von Jahren ohne solche Ausgleichung fortbestehen kann (wie in den Mery a n'schen Fällen), ist dahin zu erklären, dass hier nur ein verhältnissmässig vorübergehender Reiz wirkt, während in jenen Krankheiten ein andauernder besteht, durch allgemeine Störungen hervorgebracht.

Heidelberg, Juli 1865.

\section{Erklärung der Abbildungen auf Taf. XXV.}

Fig. 1. Schema einer Muskelfibrille. Die zwei mittleren Fleischtheilahen und die umhüllende contractile Masse a. sind in körniger Entartung begriffen.

Fig. 2. Durch unmittelbare Einwirkung des Aetzmittels auf den Faserinhalt in Detritusmasse zerfallene Muskelfaser. $350 / 1$.

Fig. 3. Bei beginnendər Entartung brüchig gewordene Muskelfaser, die eine Neigung zum Zerfall in horizontale Scheiben zeigt. $\mathbf{3 5 0 / 1}$.

Fig. 4. Muskelfaser mit vergrösserten, theilweise mit Fetttröpfchen angefüllten Muskelkörperchen. 400/1.

Fig. 5. Körniger Zerfall der Muskelfaser, mit schwachen Spuren der Fettentartung. 250/1.

Fig. 6. Vorgerücktes Stadium der Fettentartung. 250/1, 


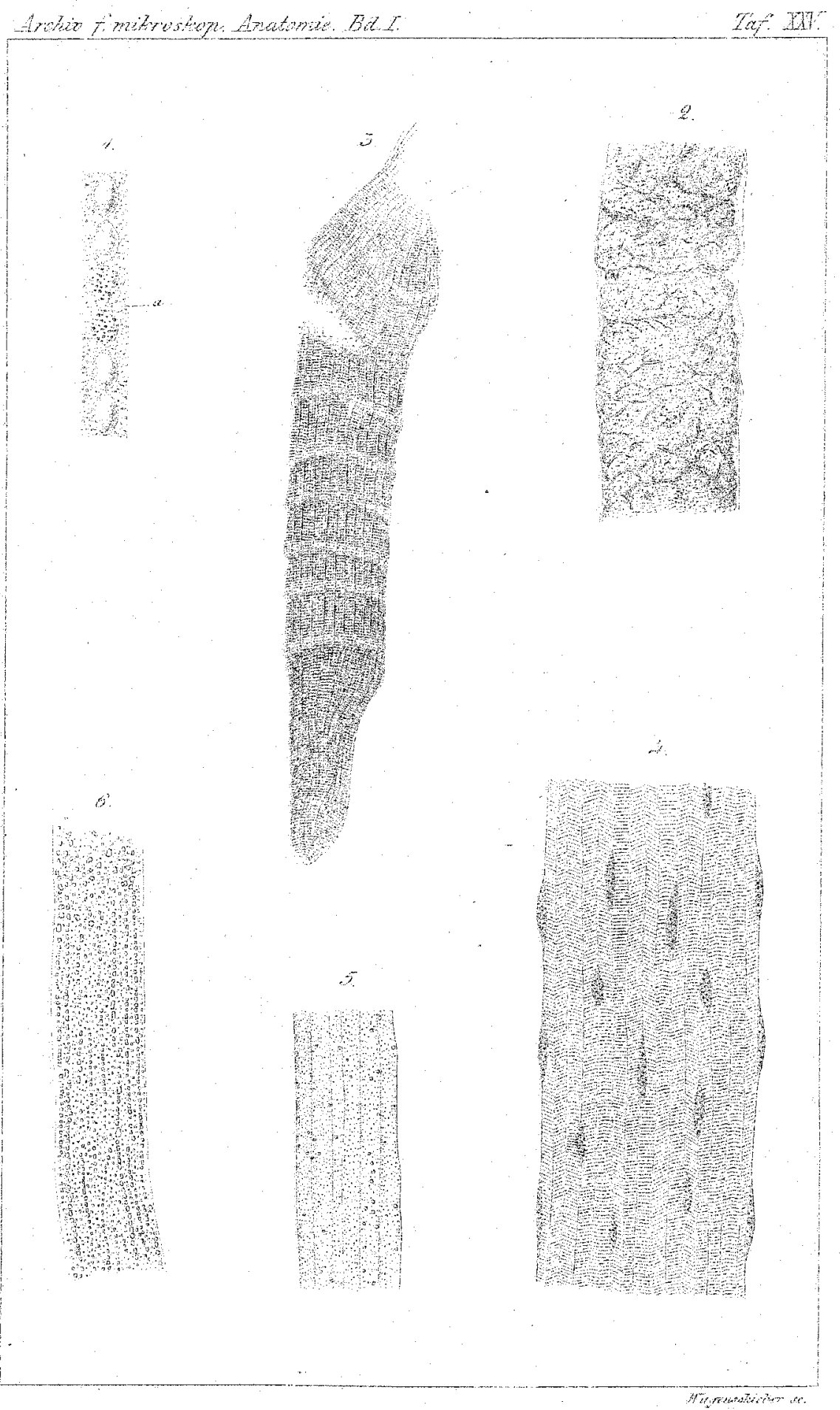

